Die Rom III-Verordnung ist ein Paradebeispiel dafür, wie schwierig Rechtsetzung in Brüssel insbesondere im Familienrecht sein kann.

Im Familienrecht ist bekanntlich Einstimmigkeit im Rat erforderlich.

Und deshalb verwundert es nicht, dass gerade die Beratungen zu Rom III überaus kompliziert waren. Denn hier waren die Unterschiede im materiellen Recht enorm. Während es in Malta damals überhaupt noch keine Scheidung gab, konnte man sich in Schweden schon nach einer Bedenkzeit von sechs Monaten scheiden lassen.

Immerhin konnten sich letztlich - im Wege der sogenannten Verstärkten Zusammenarbeit - 14 Mitgliedstaaten, darunter auch Deutschland, auf die Rom III-Verordnung einigen.

Und erfreulicherweise hat auch Litauen mittlerweile erklärt, sich der Verstärkten Zusammenarbeit anschließen zu wollen. Ich hoffe, dass weitere Mitgliedstaaten diesem Beispiel folgen werden.

Bei der Erbrechts-Verordnung war man sich lange Zeit nicht sicher, ob man überhaupt zu einem Ergebnis kommen würde und dies, obwohl hier (anders als im Familienrecht) nur eine qualifizierte Mehrheit erforderlich ist.

Ich bin froh, dass man sich letztlich aber doch noch einigen konnte. Denn die Abwicklung grenzüberschreitender Nachlässe, die immer häufiger werden, muss erleichtert werden.

Mit ihren 84 Artikeln und 83 Erwägungsgründen ist die Erbrechts-Verordnung allerdings die umfangreichste und komplexeste Verordnung der justiziellen Zusammenarbeit in Zivilsachen.

Darin kommt deutlich zum Ausdruck, dass diese Verordnung - wie das in der EU so üblich ist - aus einer Vielzahl von Kompromissen besteht.

Mit beiden Themen, Rom III und Erbrechts-Verordnung, haben Sie sich also wirklich „dicke Bretter“ vorgenommen. Und ich bin sehr gespannt, zu erfahren, wie die Rom-III-Verordnung in der Praxis angekommen ist und was Sie uns zur Umsetzung im Erbrecht mit auf den Weg geben werden.

Meine Damen und Herren,

die große Physikerin Madame Curie hat einmal gesagt: „Man merkt nie, was schon getan wurde, man sieht immer nur, was noch zu tun bleibt.“
Bis zur vollständigen gesellschaftlichen Gleichberechtigung der Geschlechter bleibt noch Einiges zu tun. Womit ich zwanglos beim Thema „Frauen in Führungspositionen“ bin.

Als erste weibliche Staatssekretärin des BMJ freut mich nicht nur, dass wir bei diesem Thema in Vorständen und Aufsichtsräten sichtlich vorankommen - auch dank Ihres massiven Einsatzes. Ich habe mich natürlich auch als sogenannte Amtschefin dieses Themas angenommen und versucht, in dieser Legislaturperiode die Vereinbarkeit von Beruf und Familie auch im Ministerium weiter zu fördern. Wir haben zurzeit über 250 verschiedene Teilzeitmodelle. Und seit 2009 hat sich die Zahl der Telearbeiter bei uns verdoppelt. Unsere Frauenquote bei den Juristen liegt schon seit längerem über 40 Prozent. Aber von 2009 bis heute haben wir den Anteil von Frauen in Führungspositionen von 31,5 Prozent auf knapp 37 Prozent steigern können.

Mit dem Personalrat habe ich eine Dienstvereinbarung über mobiles Arbeiten abgeschlossen, eine flexible Arbeitsform, die der besseren Vereinbarkeit von Privat- und Berufsleben dient. Wir haben einen Familienservice, einen Familientag, prüfen zurzeit Belegkontingente bei externen Kinderbetreuungseinrichtungen, haben uns einem Audit Beruf und Familie unterworfen und bemühen uns um familienfreundliche Fortbildungsangebote.

Und last but not least habe ich gemeinsam mit unserer Gleichstellungsbeauftragten und unserer Personalratsvorsitzenden die Dienstvereinbarung zur Beurteilung überarbeitet, um auch hier Geschlechtergerechtigkeit zu gewährleisten. Jedenfalls die von uns, die im öffentlichen Dienst sind, wissen ja wie wichtig die Beurteilung für den weiteren Berufsweg ist.

Ich habe selbst zwei Kinder und bin fest davon überzeugt, dass die Kombination all dieser Instrumente der richtige Weg ist - meines Erachtens weit besser - da möchte ich mich doch „outen“ - als Quoten.

Und nun darf ich Ihnen nicht nur einen interessanten und lebhaften Austausch, sondern vor allem eine ergebnisreiche Veranstaltung wünschen!

Vielen Dank für Ihre Aufmerksamkeit!

\title{
Grußwort zur Eröffnung des 40. Bundeskongresses des Deutschen Juristinnenbundes am 26. September 2013 in Leipzig
}

\author{
Christine Clauß \\ Staatsministerin für Soziales und Verbraucherschutz, \\ Freistaat Sachsen'
}

Sehr geehrte Damen und Herren, seit mehr als 60 Jahren besteht der Deutsche Juristinnenbund e.V. nun schon als Zusammenschluss von Juristinnen, Volkswirtinnen und Betriebswirtinnen. Ihr Verein befasst sich, wie auch mein Ministerium, mit Fragen der Sozialpolitik in allen Facetten der Arbeitsmarktpolitik sowie dem Thema Zuwanderung und häusliche Gewalt. Schwerpunkte sind dabei die Gleichberechtigung und Gleichstellung der Frau in Gesellschaft, Beruf und Familie sowie der rechtlichen Absicherung der Lebenssituation von Kindern und alten Menschen. Die gesellschaftliche Entwicklung der vergangenen Jahre führte immer wieder zu rechtspolitischen Vorschlägen, Gesetzesinitiativen und Neuregelungen in diesen Bereichen, wobei der djb stets mit seinen fachlichen Stellungnahmen und Vorschlägen gefragt war.

1 Schriftliches Grußwort. 
Der 40. Bundeskongress des djb dreht sich um das große Thema der Familie. Dabei geht es insbesondere um die vermögensrechtliche Auseinandersetzung in der Ehe- und Lebenspartnerschaft mit Blick auf eine gleichberechtigte Teilhabe von Frauen und Männern. Schwerpunkte sind das eheliche Güterrecht und das Unterhaltsrecht, das gegenwärtig stark diskutiert wird, sowie der Zusammenhang zwischen Betreuungsregelungen und Kindesunterhalt.

Für mich ist es wichtig, dass die finanziellen Rahmenbedingungen für Familien stimmen. Daher setze ich auf eine Strukturpolitik, die durch die Gestaltung familienfreundlicher Bedingungen junge Paare bei der Realisierung ihrer Kinderwünsche unterstützt.
Grundsätzlich gilt: Familienpolitik ist eine gesamtgesellschaftliche Aufgabe und muss auch als solche begriffen werden. Dabei ist für mich nicht die Frage wichtig „Was kostet uns Familie?“, sondern vielmehr „Was ist uns Familie wert?“.

Die Gleichberechtigung von Mann und Frau ist de jure erreicht, aber noch nicht de facto. Deshalb bedarf die rechtliche Absicherung der Lebenssituation von Frauen, Kindern und älteren Menschen unverändert große Anstrengungen aller gesellschaftlichen Kräfte, aber vor allem auch von Ihnen als Juristinnen. Für Ihr Engagement und Ihren Einsatz danke ich Ihnen und sage: Herzlichen Glückwunsch zu Ihrem Jubiläum.

\section{Grußwort zur Eröffnung des 40. Bundeskongresses des Deutschen Juristinnenbundes am 26. September 2013 in Leipzig}

\section{Birgit Munz}

Präsidentin des Verfassungsgerichtshofs des Freistaates Sachsen, Vizepräsidentin des Oberlandesgerichts, Dresden

Sehr geehrte Frau Präsidentin Pisal,

sehr geehrte Damen,

sehr geehrte Herren,

vielen Dank für die freundliche Einladung zur heutigen Eröffnungsveranstaltung. Ich möchte die Gelegenheit für einige Gedanken über Frauen in der Justiz, speziell in der sächsischen Justiz nutzen.

Sie haben für Ihren diesjährigen Bundeskongress eine Stadt mit großer Gerichtstradition gewählt - dieses Gebäude ist hierfür ein sinnfälliger Ausdruck. Auch über Frauen in der Justiz wird in dieser Stadt schon seit langem nachgedacht. Vor etwas mehr als 90 Jahren, im Mai 1921, fand hier in Leipzig der 4. Deutsche Richtertag statt, der sich unter anderem mit dem Thema „Die Zulassung der Frau zum Richteramt" beschäftigte ${ }^{1}$. Die damalige Debatte hierüber mutet aus heutiger Sicht skurril an: Die Frau sei aufgrund ihrer seelischen Eigenart für das Richteramt ungeeignet, da sie insbesondere während Monatsperiode, Schwangerschaft und Wechseljahren Gefühlseinflüssen unterworfen sei, die ihre sachliche Auffassung beeinträchtigten. Oder: Die Frau könne sich zwar Gesetzesbestimmungen gedächtnismäßig zu eigen machen, für die zur Rechtsprechung erforderliche produktive Geistestätigkeit seien ihr aber unüberwindliche Grenzen der Begabung gezogen. Angesichts der Schlagkraft solcher Argumente wundert es kaum, dass auf diesem Richtertag die Stimmen kein Gehör fanden, die auf die auch damals schon eindeutige Verfassungslage - nach Artikel 109 Weimarer Reichsverfassung hatten Mann und Frau grundsätzlich dieselben staatsbürgerlichen Rechte und Pflichten - hinwiesen. Die Entschließung des Richtertages lautete daher mit großer Mehrheit dahin, dass die Frau als Berufsrichterin und Schöffin nicht in Frage komme.

Wie wir wissen, hat diese Entschließung die Entwicklung ebenso wenig aufhalten können, wie die Erkenntnis, dass die Erde eine Scheibe ist: Nur ein Jahr später, im Juli 1922, wurde den Frauen der Zugang zu den Berufen der Rechtspflege eröffnet und 1927 wurde die erste Frau zur Richterin ernannt.
Und wie steht es heute um die Frauen in der Justiz? In der sächsischen Justiz waren im Jahre 2010 - aktuellere Daten liegen mir aufgrund des Erhebungszeitraumes derzeit leider noch nicht vor - von den bis zu 35 Jahre alten Richtern und Staatsanwälten 60 Prozent Frauen, insgesamt betrug der Frauenanteil in der sächsischen Justiz 43 Prozent. Deutlich anders sieht es jedoch in den Beförderungsämtern aus: Hier waren nicht einmal 30 Prozent mit Frauen besetzt. Bei den wirklichen Führungspositionen braucht man noch nicht einmal die Statistik zu bemühen. Hier sprechen die absoluten Zahlen für sich: In Sachsen gibt es keine Präsidentin eines Obergerichts, keine Landgerichts- oder Amtsgerichtspräsidentin, keine Sozialgerichtspräsidentin, keine Direktorin eines Arbeitsgerichts und keine leitende Oberstaatsanwältin. Frauen stehen lediglich zwei - von drei - Verwaltungsgerichten und sechs - von 22 - Direktorenamtsgerichten vor.

Kann man hieran etwas ändern? Ist eine Frauenquote hierfür ein geeignetes Mittel? Muss man überhaupt etwas unternehmen oder werden die jungen Kolleginnen der neuen Richtergeneration ganz automatisch mit neuem Rollenverständnis und neuem Selbstbewusstsein ihren Anteil an Führungspositionen erobern?

Wenn ich ehrlich bin, kann ich diese Fragen nicht eindeutig beantworten. Ich glaube aber, dass die Ursachen für die geringe Repräsentation von Frauen in leitenden Positionen der Justiz heute keine wesentlich anderen sind als vor zwanzig oder dreißig Jahren.

Die FAZ titelte kürzlich „Für Kita statt Quote“2 und fasste mit dieser Überschrift das Ergebnis einer im Auftrag der Zeitschrift „Emma“ erstellten Studie des Allensbach Institutes zum Stand der Gleichberechtigung in Deutschland zusammen. Diese Studie hat ergeben, dass nur knapp ein Viertel der weiblichen Befragten der Ansicht ist, dass eine Frauenquote wichtig sei, um Frauen im Beruf zu fördern. Auf der Prioritätenliste stehen vielmehr flexible Betreuungszeiten in Kindergärten, aber auch Teilzeit und flexible Arbeitszeiten für Mütter und Väter. Besonders erwähnenswert ist in diesem Zusammenhang, dass 68 Prozent aller Befragten

1 Vgl. hierzu das Protokoll vom 4. Deutschen Richtertag zum Thema „Die Zulassung der Frau zum Richteramt“, Deutsche Richterzeitung 1921, S. 196 ff.

2 Frankfurter Allgemeine vom 27. August 2013. 